\title{
Electron tunneling through graphene-based double barriers driven by a periodic potential
}

\author{
H. P. Ojeda-Collado and C. Rodríguez-Castellanos \\ Department of Theoretical Physics, Faculty of Physics University of Havana, \\ San Lázaro y L, Vedado, La Habana, Cuba, CP 10400
}

(Dated: July 26, 2018)

\begin{abstract}
Photon-assisted charge transport through a double barrier structure under a time periodic field in graphene is studied. Within the framework of the Floquet formalism and using the transfer matrix method, the transmission probabilities for the central band and sidebands are calculated. A critical phase difference between the harmonic potentials at the barriers, which cancels transmission through the inelastic sidebands due to quantum interference is found. This phenomenon could be of help to design graphene based filters and high-frequency radiation detectors. Quenching of resonant tunneling by the harmonic field applied to the barriers or the well is also discussed.
\end{abstract}

Quantum transport in periodically driven mesoscopic systems is an important subject not only of academic value but also for device applications. The interest on time-dependent excitations by electromagnetic fields has been increasing and many interesting phenomena have been investigated [1, 2]. Early studies of Dayem and Martin 3] provided the evidence of photon assisted tunneling (PAT) in experiments on superconducting films under microwave fields, and subsequently Tien and Gordon theoretically justified this observation [4]. After this, electron transport through various types of timeoscillating potential regions has been studied in semiconductor nanostructures [5-8] aiming to the fabrication of new devices.

A few years ago, graphene, a two dimensional honeycomb crystal of carbon atoms, was fabricated by Novoselov et al. [9], leading to increasing attention to both the fundamental physics and potential applications of the new material. In graphene, low-energy quasiparticle excitations near the edges of the hexagonal Brillouin zone (Dirac points) obey a gapless linear dispersion law, and their motion can be described by a two-dimensional Dirac equation for masless particles. The presence of such Dirac-like quasiparticles leads to Klein tunneling and other unusual electronic properties 9 -14]. For graphenebased PAT devices it is essential to consider transport of charge carriers in graphene through time-harmonic potentials. PAT through single barrier in monolayer graphene have been discussed in Ref. [15]. This letter is an extension for double barrier structures (DBS) taking into account quantum interference between photon assisted processes. At normal incidence PAT is analyzed aiming to get a direct insight into the quantum interference and analytical condition for the suppression of the transmission through the inelastic sidebands is obtained. We also find that quantum interference makes a significant contribution to the total transmission probability at non-normal incidence and could be useful in graphene-based PAT devices.

A single electron transmitting through a monolayer graphene-based double barrier driven by a harmonic potential is considered. This structure can be fabricated by

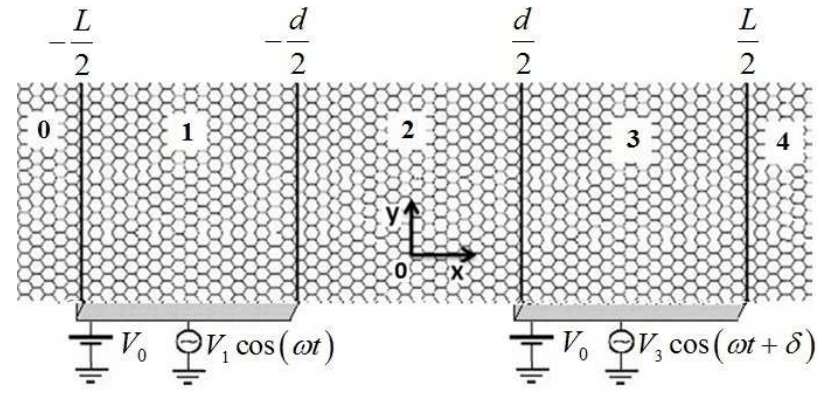

FIG. 1: Schematic oscillating potential of the DBS in graphene.

applying a local top gate voltage and a small ac signal to graphene. At low energy, and close to the Dirac points ( $\mathrm{K}$ and $\mathrm{K}$ ') electrons are described by a massless Dirac Hamiltonian:

$$
\hat{H}=v_{f} \hat{\vec{\sigma}} \cdot \hat{\vec{p}}+\hat{V}(x, t)
$$

with

$$
\begin{aligned}
\hat{V}(x, t) & =\hat{I}\left[V_{0}+V(x) \cos (\omega t+\delta \vartheta(x))\right] \\
& \times \vartheta\left(|x|-\frac{d}{2}\right) \vartheta\left(\frac{L}{2}-|x|\right)
\end{aligned}
$$

where the Fermi velocity $v_{f} \approx 10^{6} \mathrm{~m} / \mathrm{s}, \hat{\vec{\sigma}}=\left(\hat{\sigma_{x}}, \hat{\sigma_{y}}\right)$ is the $2 \mathrm{D}$ vector of Pauli matrices and $\overrightarrow{\vec{p}} \rightarrow-i \hbar \vec{\nabla}$ is the $2 \mathrm{D}$ momentum operator. The barriers of height $V_{0}$ are oscillating with frequency $\omega$, phase difference $\delta$, and different amplitude: $V(x)=V_{1}$ if $x<0$ or $V(x)=V_{3}$ for $x>0$. This simple device is shown schematically in Fig. 1. The barriers and well widths are $L_{1}=(L-d) / 2$ and $L_{2}=d$ respectively, $\vartheta(x)$ is the Heaviside function and $\hat{I}$ is unit matrix. The DBS is infinite and homogeneous along the y-direction, resulting in the conservation of the y-component of momentum. Incident electrons propagate from left and pass five regions denoted by $0,1,2,3$, 4. The solution of Dirac-like equation with hamiltonian 
(11) for given quasienergy $E$ and y-component of momentum $k_{y}$ can be written as a Floquet state:

$$
\begin{aligned}
\psi^{r}(x, y)= & \frac{e^{i k_{y} y}}{\sqrt{2}} \sum_{m, n=-\infty}^{+\infty}\left[a_{n}^{r}\left(\begin{array}{c}
1 \\
s_{n}^{r} e^{i \varphi_{n}^{r}}
\end{array}\right) e^{i k_{n}^{r}\left(x-x_{r}\right)}\right. \\
& \left.+b_{n}^{r}\left(\begin{array}{c}
1 \\
-s_{n}^{r} e^{-i \varphi_{n}^{r}}
\end{array}\right) e^{-i k_{n}^{r}\left(x-x_{r}\right)}\right] \\
& \times J_{m-n}\left(\frac{V_{r}}{\hbar \omega}\right) e^{-i(m-n) \delta_{r}} e^{-i(E+m \hbar \omega) t / \hbar}
\end{aligned}
$$

where the script $r=0,1,2,3,4$ indicates the region, $x_{r}$ are the coordinates of the boundaries: $x_{0} \equiv x_{1}=-L / 2$, $x_{2}=-d / 2, x_{3}=d / 2, x_{4}=L / 2$, (see Fig. 1) and $J_{n}$ is the Bessel function of the first kind. In barrier regions $(r=1,3)$ :

$$
\begin{gathered}
s_{n}^{r}=s_{n}^{\prime}=\operatorname{sgn}\left(E+n \hbar \omega-V_{0}\right), \\
k_{n}^{r}=q_{n}=\sqrt{\left(\frac{E-V_{0}+n \hbar \omega}{\hbar v_{f}}\right)^{2}-k_{y}^{2}}, \\
\varphi_{n}^{r}=\theta_{n}=\arctan \left(k_{y} / q_{n}\right) .
\end{gathered}
$$

On the other hand for $r=0,2,4$ :

$$
\begin{gathered}
s_{n}^{r}=s_{n}=\operatorname{sgn}(E+n \hbar \omega), \\
k_{n}^{r}=k_{n}=\sqrt{\left(\frac{E+n \hbar \omega}{\hbar v_{f}}\right)^{2}-k_{y}^{2}} \\
\varphi_{n}^{r}=\phi_{n}=\arctan \left(k_{y} / k_{n}\right)
\end{gathered}
$$

and $J_{m-n}\left(V_{r} / \hbar \omega\right)=\delta_{m, n}$ because in this case, the modulation amplitude is $V_{r}=0$. The phase difference $\delta_{r}=\delta$ only for $r=3$, elsewhere is zero. As Dirac electrons pass through a region subjected to time-harmonic potentials, transitions from the central band to sidebands (channels) at energies $E \pm m \hbar \omega(m=0,1,2, \ldots)$ occur as electrons exchange energy quanta with the oscillating field.

The wave function is continuous at the boundaries, and the continuity condition can be expressed as:

$$
\left(\begin{array}{l}
\mathbb{A}^{0} \\
\mathbb{B}^{0}
\end{array}\right)=\left(\begin{array}{ll}
\mathbb{K}_{11} & \mathbb{K}_{12} \\
\mathbb{K}_{21} & \mathbb{K}_{22}
\end{array}\right)\left(\begin{array}{l}
\mathbb{A}^{4} \\
\mathbb{B}^{4}
\end{array}\right)=\mathbb{K}\left(\begin{array}{l}
\mathbb{A}^{4} \\
\mathbb{B}^{4}
\end{array}\right)
$$

where the total transfer matrix $\mathbb{K}=\mathbb{K}(0,1) \cdot \mathbb{K}(1,2)$. $\mathbb{K}(2,3) \cdot \mathbb{K}(3,4)$, and $\mathbb{K}(r, r+1)(r=0,1,2,3)$ are transfer matrices that couple the wave function in the $r$-th region to the wave function in the $(r+1)$-th region. We assume an electron propagating from left to right, then, $\mathbb{A}^{0}=\left\{\delta_{n, 0}\right\}$ and $\mathbb{B}^{4}$ is the null vector, whereas $\mathbb{A}^{4}=\left\{a_{n}^{4}\right\}$ and $\mathbb{B}^{0}=\left\{b_{n}^{0}\right\}$ are the coefficient vectors of transmitting waves and of reflecting waves respectively. The total transmission probability for quasienergy $E$ is $T=\sum_{n=-\infty}^{+\infty} T_{n}$, where

$$
T_{n}=\frac{s_{n} \cos \left(\phi_{n}\right)}{s_{0} \cos \left(\phi_{0}\right)}\left|a_{n}^{4}\right|^{2}
$$
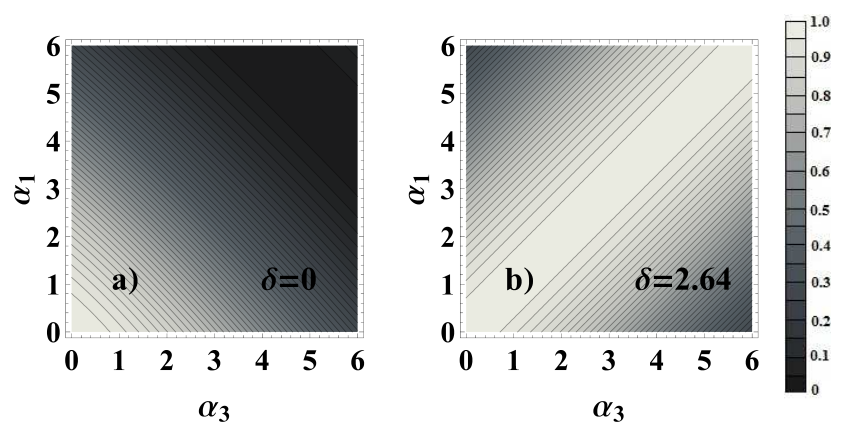

FIG. 2: Transmission probability for central band as a function of $\alpha_{1}$ and $\alpha_{3}$ at $\phi_{0}=0$ for two value of $\delta$.

is the probability of the scattering for an electron with incident quasienergy $E$ in region 0 into the sideband with quasienergy $E+n \hbar \omega$ in region 4 . The minimum number $N$ of sidebands that need to be considered is determined by the strength of the oscillation, $N>\max \left(V_{1} / \hbar \omega, V_{3} / \hbar \omega\right)$ 8, 15], and the infinite series for $T$ can be truncated to consider a finite number of terms starting from $-N$ up to $N$.

Numerical calculations have been made for barriers and well of the same width: $d=50 \mathrm{~nm}, L=150 \mathrm{~nm}$, $V_{0}=200 \mathrm{meV}, \omega=5 \times 10^{12} \mathrm{~Hz}$ and $\lambda=50 \mathrm{~nm}$ for the wavelength of incident electrons.

Normal incidence.

The dependence of the transmission probability for the central band $\left(T_{0}\right)$ on $\alpha_{1}=V_{1} / \hbar \omega$ and $\alpha_{3}=V_{3} / \hbar \omega$ for normally incident electrons is shown in Fig. 2 for two values of the phase difference $\delta$. For small values of $\alpha_{1}$ and $\alpha_{3}$ the transmission is perfect and take place only through the elastic band, because the oscillating barrier can be treated approximated by a static one, and for normal incidence, perfect transmission (Klein tunneling) through static single [13] and double barriers has been obtained [16]. With increasing $\alpha_{1}$ and $\alpha_{3}$, higher and lower sidebands become important since electrons can exchange a large number of photons with the time-periodic field, decreasing the transmission probability through the central band $\left(T_{0}\right)$ because these probabilities are now spread. However, numerical calculations show the existence of a critical value $\delta_{c}=2.64$ of the phase difference, at which the transmission probability is exclusively through the central band even for large $\alpha_{1}$ and $\alpha_{3}$ whenever $\left|\alpha_{1}-\alpha_{3}\right|<1$. Thus, the quasienergy of the transmitted electrons is sensitive to the phase difference, because tuning $\alpha_{1}, \alpha_{3}$ and $\delta$ could eliminate inelastic sidebands doing the function of an energy filter.

In order to understand the phenomenon behind this result, an analytical expression for $T_{0}$ at normal incidence is derived. Let us take the barriers with the same modulation amplitudes $\alpha_{1}=\alpha_{3}$. To simplify our calculation and get some direct insight into the interference effect [17], we take $\alpha_{1}$ as a perturbation, and include the lowest order corrections up to $\left(V_{1} / \hbar \omega\right)^{2}$, retaining only the terms corresponding to the central band and first sidebands. 
Hence, the probability for tunneling through the central band can be written as:

$$
T_{0}=\left|a_{0}^{4}\right|^{2}=\frac{\gamma+\frac{1}{8}\left(\frac{\hbar \omega}{V_{1}}\right)^{2}}{1+\frac{1}{8}\left(\frac{\hbar \omega}{V_{1}}\right)^{2}} .
$$

The term $\gamma=\left(z p^{\star}+z^{\star} p\right) / 8$ contains all the interference effects. The complex values $z$ and $p$ are given by:

$$
\begin{gathered}
z=2 e^{i\left(\delta+k_{0} L_{2}+L_{1}\left(q_{-1}+q_{0}+q_{1}\right)\right)}\left[e^{i q_{-1} L_{1}}+e^{i q_{1} L_{1}}\right] \\
+e^{i\left(k_{-1} L_{2}+2 L_{1}\left(q_{-1}+q_{0}+q_{1}\right)\right)}\left[e^{-2 i q_{-1} L_{1}}+e^{-2 i q_{0} L_{1}}\right] \\
-2 e^{i\left(k_{-1} L_{2}+L_{1}\left(q_{-1}+q_{0}+2 q_{1}\right)\right)} \\
+e^{i\left(2 \delta+k_{1} L_{2}+2 L_{1}\left(q_{-1}+q_{0}+q_{1}\right)\right)}\left[e^{-2 i q_{1} L_{1}}+e^{-2 i q_{0} L_{1}}\right] \\
-2 e^{i\left(2 \delta+k_{1} L_{2}+L_{1}\left(2 q_{-1}+q_{0}+q_{1}\right)\right)}
\end{gathered}
$$

and

$$
p=e^{i\left(\delta+k_{0} L_{2}+2 L_{1}\left(q_{-1}+q_{1}\right)\right)} .
$$

Thus, when $\gamma=1$ the suppression of inelastic sidebands is obtained under the condition:

$$
\delta_{c} \pm\left(k_{ \pm 1}-k_{0}\right) L_{2} \pm\left(q_{0}-q_{ \pm 1}\right) L_{1}=\pi .
$$

The left-hand side of (111) is the phase difference between two amplitudes. The first one corresponds to particles that propagate through the DBS and absorb (emit) the energy quantum $\hbar \omega$ at the first barrier, whereas the second one corresponds to the same propagation with absorption (emission) at the second barrier. Noteworthy that $\pm\left(k_{ \pm 1}-k_{0}\right) L_{2}$, is the phase difference between the amplitude corresponding to particles that absorb $(+)$ or emit (-) a photon close to the left barrier and traverse the well with energy $E \pm \hbar \omega$ and the amplitude corresponding to particles that absorb (emit) a photon close to the right barrier and traverse the well with energy $E$. In the same way, $\pm\left(q_{0}-q_{ \pm 1}\right) L_{1}$ is the spatial phase difference between the above mentioned amplitudes through the barrier region. Therefore, the suppression of transmission through the inelastic sidebands is due to destructive quantum interference between these two amplitudes. At normal incidence $k_{n}-k_{n-1}=q_{n-1}-q_{n}=\omega / v_{f}$ and $k_{-n}-k_{-(n-1)}=q_{-(n-1)}-q_{-n}=-\omega / v_{f}$ with $(n=1,2, \ldots)$, result from the gapless and linear electron dispersion law near the Fermi energy. Therefore, we can also write the expression (11) in general form as:

$$
\delta_{c}+L_{2}+L_{1}=\pi,
$$

where $L_{1}$ and $L_{2}$ are expressed in units of $v_{f} / \omega$ and the critical phase difference depends only on barrier and well width. Thus, Eq.(12) indicates a simultaneous destructive interference of contiguos channels at $\phi_{0}=0$. Fig. 3a) shows the transmission probability through the elastic channel as a function of $L_{1}$ and $\delta$ at $\alpha_{1}=7$ calculated after Eq.(7). The straight line in this figure is Eq.(12) at $L_{2}=0.25$. In panel b) only the region where $1-T_{0}<0.005$ for different well widths is shown. The analytical result presented as condition (12) remains valid even in the regime $\alpha_{1} \gg 1$, when a large number of channels coexist, and show excellent agreement with numerical solutions in Fig. 3. Thus, quantum interference between first order process is the physical basis behind Eq.(12). Now, it is clearly seen why $\left|\alpha_{1}-\alpha_{3}\right|<1$ is necessary for the suppression of the transmission through inelastic sidebands. The same amplitude of probability for the process of absorption (emission) on both barrier region is required to achieve destructive interference. It should be pointed out that, at normal incidence, perfect total transmission, reported for static [13] and oscillating [15] single barriers persists for harmonically driven DBS regardless of interference effects.

\section{Oblique incidence.}

Now, resonant tunneling through DBS at oblique incidence under time periodic field is investigated. Due to the existence of evanescent modes inside the static barrier [14], the transmission, as a function of the incident energy, has a gap which can be tuned changing the height of the barrier and/or the angle of incidence [18]. Because of coupling of evanescent states in the barrier with resonant states in the well, several resonance peaks with a unity value appear in the transmission gap [19]. In the following, the influence of oscillation amplitudes and interference effects on resonant tunneling is studied. Fig. 4 shows total transmission probability as a function of the energy of incident electrons at $\phi_{0}=\pi / 18$ for different amplitudes of the oscillating field and $\delta=0$. In Fig. 4a) the transmission probability for the static double barrier is shown as it corresponds to $\alpha_{1}=\alpha_{3}=0$, which was previously obtained. Resonant peaks are narrow and could have very important applications in high-speed devices based on graphene as has been suggested in Ref. [19]. Considering the effect of PAT, some satellite peaks appear on both sides of the two main resonant peaks, and as the amplitude of the oscillating field increases, satellite peaks move away from the two main resonant peaks due to emission or absorption of a greater number of photons. Coupling between evanescent wave inside the barriers and propagating wave inside the well occur for several energy values. The positions of the satellite peaks are
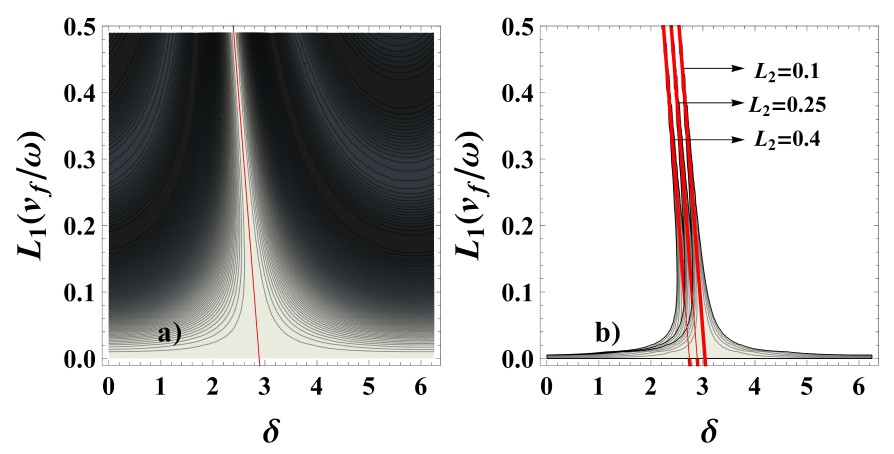

FIG. 3: (Color online) $T_{0}$ as a function of $L_{1}$ and $\delta$ at $\phi_{0}=0$, $\alpha_{1}=7$ and a) $L_{2}=0.25 \mathrm{~b}$ ) well widths; $0.1,0.25$ and 0.4 . The red line is (12) at $L_{2}$ fixed. 

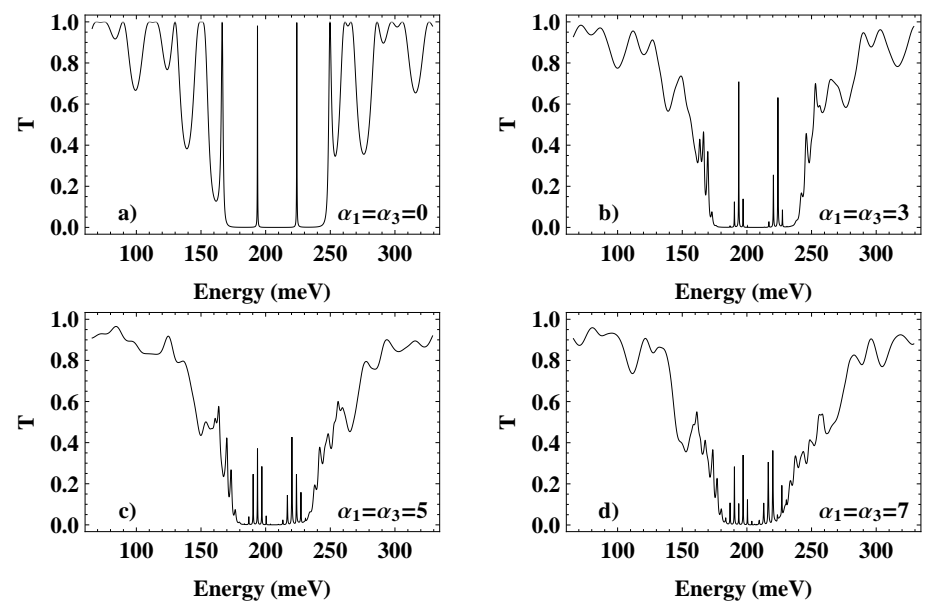

FIG. 4: Total transmission probability on incident energy at $\phi_{0}=\pi / 18$ and $\delta=0$ for several value of the modulation amplitude.

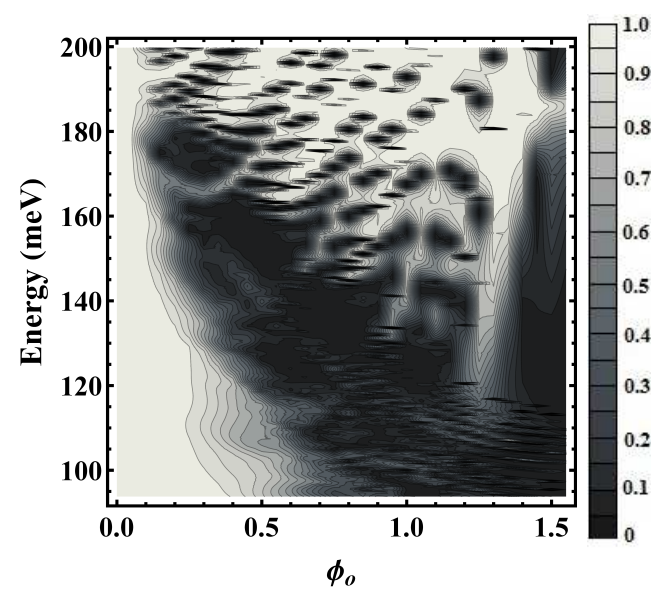

FIG. 5: $T_{0} / T$ as a function of incident energy and angle for $\alpha_{1}=\alpha_{3}=7$ and $\delta=2.64$.

$E_{1,2} \pm n \hbar \omega$ where $E_{1,2}$ are the positions of the main resonant peaks. At $\alpha_{1}=\alpha_{3}=3$ only two main peaks with few small satellites are obtained. However, at high amplitude modulation $\left(\alpha_{1}=\alpha_{3}=7\right)$, the two main peaks lose its dominance and the intensities of main peaks and satellite peaks are reversed. Therefore, strong quenching of resonant transmission with increasing amplitude of oscillating field is found as shown in panel d) of Fig. 4.

For non-normal incidence $k_{ \pm n}-k_{ \pm(n-1)}$ and $q_{ \pm(n-1)}-$ $q_{ \pm n}$ depend on $E \pm n \hbar \omega, V_{0}$ and $\phi_{0}$. Then, it is not possible to determine analytically a critical phase difference which cancels simultaneously all contiguos channels. However, suppression of inelastic sidebands at $\delta=2.64$ is obtained numerically not only for normal incidence but $\left(T_{0}>0.95 T\right)$ for different values of the incident energy and incident angle at $\alpha_{1}=\alpha_{3}=7$ as shown in Fig. 5. Thus, destructive interference effects are robust, since the phase diagram ( $E$ vs $\left.\phi_{0}\right)$ in Fig. 5 exhibits

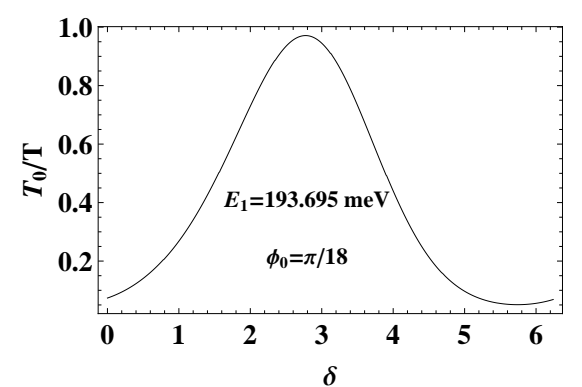

FIG. 6: $T_{0} / T$ as a function of phase difference at $\alpha_{1}=\alpha_{3}=7$.

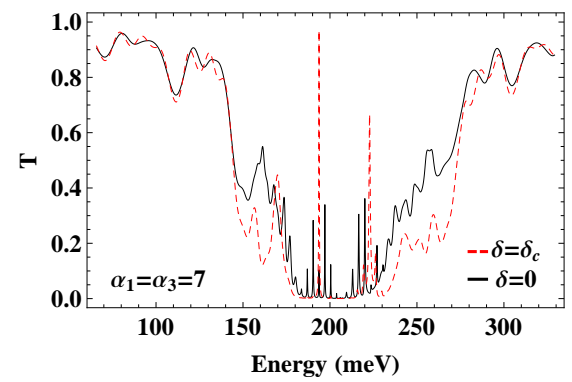

FIG. 7: (Color online) $T$ as a function of incident energy at $\phi_{0}=\pi / 18$ for $\delta=0$ (black line) and $\delta=2.76$ (dashed red line).

many white zones at oblique incidence, corresponding to strong quenching of the transmission through inelastic sidebands. These zones change with phase difference and become highly anisotropic due to the chiral nature of quasiparticles in graphene. Fig. 6 shows $T_{0} / T$ as a function of $\delta$ for incident energy corresponding to first main resonant peaks $\left(E_{1}=193.695 \mathrm{meV}\right)$ and $\phi_{0}=\pi / 18$. Note that transmission is practically only through central band for $\delta=2.76$ and for other phase differences inelastic sidebands will become more important. Thus, changing $\delta$ we shall observe radical influence in the resonant features. Fig. 7 shows total transmission probability as a function of energy at $\phi_{0}=\pi / 18$ and $\alpha_{1}=\alpha_{3}=7$ for two values of the phase difference. Adjusting the value of $\delta$ resonant transmission can be controlled. For $\delta=2.76$ satellite peaks are strongly suppressed and almost perfect transmission is obtained for $E_{1}=193.695 \mathrm{meV}$ whereas at $\delta=0$ a large number of sidebands coexist and resonant tunneling practically disappears. Thus, quantum interference makes a considerable contribution to total transmission at non-normal incidence.

\section{Oscillating well.}

In addition, total transmission probability has been also calculated when the harmonic potential is applied at the well instead of the barrier. In Fig. 8 PAT in a driven DBS with oscillating quantum well (panel b) and oscillating quantum barriers (panel a), are compared. We observe that the driving field with amplitude $3 \hbar \omega$ is more effective in quenching of resonant tunneling when applied to the well. It occurs because, for the incident energy in the 

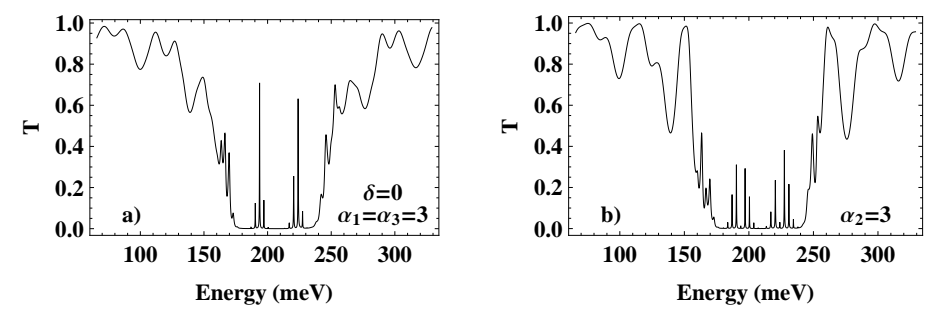

FIG. 8: $T$ as a function of incident energy at $\phi_{0}=\pi / 18$ for a) oscillating quantum barriers and b) oscillating quantum well.

gap region $\left(V_{0}-\hbar k_{y} v_{f}<E<V_{0}+\hbar k_{y} v_{f}\right)$, the evanescent modes appear inside the barriers and the electrons reflect back and forth several rounds in the well. Thus, for oscillating quantum well the electrons have enough time to interact with the driving field contrary to oscillating quantum barriers. Consequently, resonant peaks are more strongly quenched in driven well.

In summary, we have carried out a study of electron PAT through graphene-based symmetric double barriers driven by a periodic potential. Barriers oscillate with the same frequency $\omega$, different amplitudes and phase difference $\delta$. The time-periodic electromagnetic field generates additional sidebands at energies $E \pm n \hbar \omega$ in the transmis- sion probability due to photon absorption or emission. At normal incidence, perfect total transmission probability (Klein tunneling) persist for harmonically driven DBS. A critical phase difference is found such that, total suppression of inelastic sidebands due to destructive interference between waves is obtained. A condition for the simultaneous cancellation of contiguos channels as a result of linear dispersion law, is derived for small amplitude modulation and is valid even in the regime $\left(\alpha_{1}=\alpha_{3} \gg 1\right)$. Thus, energy of the transmitted electrons is sensitive to phase difference and inelastic sidebands can be removed by tuning structural parameters of the DBS according to Eq.(12). For oblique incidence, suppression of inelastic sidebands occurs and destructive interference plays a fundamental role in the total transmission probability. Resonant transmission may be regulated, as resonant tunneling is quenched at $\delta=0$ and recovered for certain phase difference. Moreover, quenching of resonant transmission in driven well is more drastic than driven barriers. In conclusion, we have shown that quantum interference has an important effect on quasiparticles tunneling through a time-dependent graphene-based double barrier. This phenomenon has potential applications in graphene-based electronic devices such as energy filters and high-frequency radiation detectors.
[1] F. Grossmann, T. Dittrich, P. Jung, P. Hänggi, Phys. Rev. Lett. 67, 516 (1991)

[2] M. Moskalets and M. Büttiker, Phys. Rev. B 66, 035306 (2002)

[3] A. H. Dayem and R. J. Martin, Phys. Rev. Lett. 8, 246 (1962)

[4] P. K. Tien and J. P. Gordon, Phys. Rev. 129, 647 (1963)

[5] M. Wagner, Phys. Rev. B 49, 16544 (1994)

[6] M. Wagner, Phys. Rev. A 51, 798 (1995)

[7] G. P. Berman, E. N. Bulgakov, D. K. Campbell, and A. F. Sadreev, Physica B 225, 1 (1996)

[8] W. Li y L. E. Reichl, Phys. Rev. B 60, 15732 (1999)

[9] K.S. Novoselov, A.K. Geim, S.V. Morozov, D. Jiang, Y. Zhang, S.V. Dubonos, I.V. Gregorieva, and A.A. Firsov, Science 306, 666 (2004)

[10] E. McCann and V. I. Falko, Phys. Rev. Lett. 96, 086805 (2006)
[11] Y. Zhang, J. P. Small, M. E. S. Amori, and P. Kim, Phys. Rev. Lett. 94, 176803 (2005)

[12] O. Klein, Z. Phys. 53, 157 (1929)

[13] M. I. Katsnelson, K. S. Novoselov, and A. K. Geim, Nat. Phys. 2, 620 (2006)

[14] J. M. Pereira, Jr., V. Mlinar, F. M. Peeters and P. Vasilopoulos, Phys. Rev. B 74, 045424 (2006)

[15] M. Ahsan Zeb, K. Sabeeh, M.Tahir, Phys. Rev. B 78, 165420 (2008)

[16] C. Bai and X. Zhang, Phys. Rev. B 76, 075430 (2007)

[17] C. Zhang and N. Tzoar, Appl. Phys. Lett. 53, 1982 (1988)

[18] X. Chen and J. W. Tao, Appl. Phys. Lett. 94, 262102 (2009)

[19] L. Sun and Y. Guo, J. Appl. Phys. 109, 123719 (2011) 